\title{
Primary sarcomatoid urothelial carcinoma of the ureter: a case report and review of the literature
}

\author{
Yixiang Wang ${ }^{\dagger}$, Hanchao Liu ${ }^{\dagger}$ and Ping Wang ${ }^{*}$
}

\begin{abstract}
Background: Sarcomatoid urothelial carcinoma is a very dangerous malignant tumour derived from the epithelium. Primary sarcomatoid carcinoma of the ureter is extremely rare in clinical practice. The prognosis of this kind of disease is really poor, and there is still not a diagnosis standard in the world.

Case presentation: An 82-year-old female patient who had intermittent waist pain without any other symptoms, had diagnosed as urothelial cancer on computerised tomography urography. Considering the patient's age and quality of life, we made a preserving kidneys resection of the local tumour. The tumour was composed of sarcomatous and carcinomatous elements, and immunohistochemical examination showed that tumour cells were positive for cytokeratin, epithelial membrane antigen, vimentin, and GATA3 markers. There were no complications after 1-hour surgery. After 3 months, there was no signs of recurrence and metastasis.

Conclusion: This case was a patient with sacomatoid urothelial carcinoma. Through a transurethral resection with laser, the patient recovered well, and there was no sign of any recurrence of the tumour after 3 months. With the development of technology and science, more and more cancerous patients' living quality and survival rate were improved. Maybe it is essential for urologists and scientists to entirely understand the characteristics of the sarcomatoid urothelial carcinoma and make a better clinical guideline.
\end{abstract}

Keywords: Sarcomatoid urothelial carcinoma, Ureter, Clinical treatment

\section{Background}

Sarcomatoid urothelial carcinoma is a very dangerous malignant tumour derived from the epithelium; its cells which are sarcomatoid differentiation can occur in any cancerous parts and more common in the respiratory system [1]. Primary sarcomatoid carcinoma of the ureter is extremely rare in clinical practice. This article reported a case of primary sarcomatoid urothelial carcinoma of the ureter and the clinical treatment. According to the best of our knowledge, sarcomatoid urothelial carcinoma occurring in the urinary system is very rare, especially in the ureter. In the urinary bladder, it only accounts for 0.1 to $0.3 \%$ of carcinomas [2]. And only 24 cases of the ureteral sarcomatoid urothelial

\footnotetext{
* Correspondence: cmu4h-wp@126.com

${ }^{\dagger}$ Equal contributors

Department of Urology, The Fourth Hospital Affiliated to China Medical University, Chongshan East street, Shenyang, Liaoning 110032, People's Republic of China
}

carcinoma have been reported in the PubMed database [3]. Then, 11 adrenal sarcomatoid carcinoma cases have been reported from 1987 to 2016 [4]. The present case revealed a primary sarcomatoid urothelial carcinoma, pathology features and clinical treatments.

\section{Case presentation}

An 82-year-old female patient presenting with intermittent pain in the left flank was accepted by The Fourth Hospital Affiliated to China Medical University (LiaoNing, China) on April 19, 2017.On physical examination, the patient had no fever with a pulse rate of 88 beats/min and a blood pressure of 130/80 mmHg. There was mild left upper quadrant tenderness to palpation. She had no significant past medical or surgical history of note. She had a history of hypertension for 10 years. Laboratory examinations showed the following: white blood cell count, $4.70 \times 109 / 1$ (normal range, 4.0-10. $0 \times 109 / 1$ ); red blood cell count, $4.06 \times 1012 / 1$ (normal range, 3.5-5.0 × 1012/l); haemoglobin level, $130 \mathrm{~g} / \mathrm{l}$ (normal range, 
110-150 g/l); red blood cells in urine, 1.6/HPF; and white blood cell in urine, 4.1/HPF. Cytological examination of the urine revealed no urothelial carcinoma, squamous cell carcinoma, or small cell carcinoma components. A computerised tomography (CT) urography demonstrated that a $9 \times 7 \mathrm{~mm}$ irregular mass $(31 \mathrm{HU})$ was located in the lower ureter (Fig. 1a), which led to the dilatation of the proximal ureter and renal pelvis. During the nephrographic phase, the mass $(82 \mathrm{HU})$ had a significant contrast enhancement (Fig. 1b). Chest and abdomen computed tomography showed no evidence of distant visceral metastases. These led to suspicion of lower ureteral tumour.

Trans-urethral resection of the tumour was carried out on April 27, 2017; due to the age and life quality of the patient, the ureter was retained. The tumour with a size of 1 . $0 \mathrm{~cm}$ in the lower part of the left lower ureter was removed by a $2 \mu \mathrm{m}$ holmium laser and sent to the pathology department. Simultaneously, we used laser to cut the suspicious mucosa around the tumour and then indwelled double-J stent to prevent ureteral obstruction. Surgical samples were formalin-fixed, paraffin-embedded, and cut into $4 \mu \mathrm{m}$ thick sections for the histological examination with hematoxylin-eosin stain and immunohistochemical procedures against epithelial membrane antigen (EMA), cytokeratin (CK), vimentin, desmin, carcinoembryonic antigen (CEA), cytokeratin high molecular weight (CKH), cytokeratins 20, S100, P63, GATA3, and Ki-67 (Table 1). The histopathological findings suggested that the tumour had a biphasic appearance with an epithelial component represented by areas of urothelial high grade carcinomas and a sarcomatoid component (Fig. 2). Immunohistochemical analysis indicated positivity of the tumour cells for vimentin, CK, EMA, CEA, CKH, GATA3, and P63 (Fig. 3a, b, c, d, e, f) and negativity for Desmin, S-100, and CK20. High proliferation index (40\%) was documented against Ki-67 staining (Fig. 3g), indicating the malignant nature of the lesion. Based on the histological and immunohistochemical findings, sarcomatoid urothelial carcinoma was diagnosed. Due to patient and families rejected
Table 1 The results of the antibody panel

\begin{tabular}{llllll}
\hline Antigen & Source & Clone & Pretreatment & CC & SRC \\
\hline CK & KIT-0009 & AE1/AE3 & Trypsin & ++ & + \\
CEA & MAB-0043 & ZC23 & None & + & + \\
VIM & MAB-0735 & MX034 & PC-C & + & ++ \\
GATA 3 & MAB-0695 & L50-823 & EDTA & + & + \\
CKH & KIT-0020 & 34ßE12 & PC-C & ++ & + \\
P63 & MAB-0694 & MX013 & EDTA & + & - \\
EMA & KIT-0011 & E29 & None & ++ & + \\
Desmin & KIT-0023 & D33 & None & - & - \\
S-100 & KIT-0007 & 4C4.9 & PC-C & - & - \\
CK20 & MAB-0669 & MX003 & PC-C & - & - \\
Ki-67 & KIT-0005 & MIB-1 & PC-C & $\uparrow$ & $\uparrow$ \\
\hline
\end{tabular}

+: weak, ++: strong, -: negative, $\uparrow:$ high proliferative activity. Trypsin: sections preheated with $0.1 \%$ trypsin (sigma) for $3 \mathrm{~min}$ at $37{ }^{\circ} \mathrm{C}$. EDTA: sections preheated with ethylenediaminetetraacetic acid. PC-C: sections preheated in a conventional pressure cooker for $90 \mathrm{~s}$ in a citrate buffer solution

CK cytokeratin, CEA carcinoembryonic antigen, VIM vimentin, $C K H$ cytokeratin high molecular weight, EMA epithelial membrane antigen, $C C$ carcinomatous component, SRC sarcomatoid component

the adjuvant therapy, no postoperative radiotherapy or chemotherapy was performed. In July of this year, another ureterscope was carried to reexamine the situation where the tumour grew. It turned out to be good and there was no sign of any recurrence of the tumour.

\section{Discussion}

The urothelial carcinoma is the most common type of urinary cancers. However, sarcomatoid urothelial carcinoma is a highly malignant and rare form of urothelial carcinoma. And the histogenesis of sarcomatoid urothelial carcinoma is still a controversy issue. Two major hypotheses were presented. Some scientists consider that this type of tumour represents a collision tumour which is composed of two independent but simultaneously occurring epithelial and mesenchymal monoclonal neoplasms; others suggest that this carcinoma has a same clonal origin with branching differentiation into both components [5]. Most of the

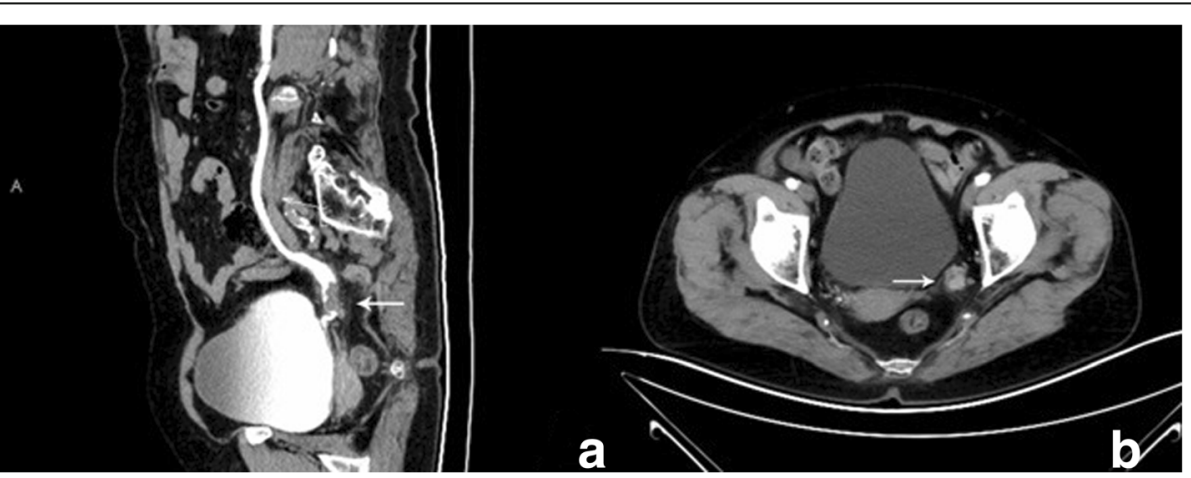

Fig. 1 a Computed tomography urography shows an irregular mass in the left lower ureter. $\mathbf{b}$ The mass had a significant contrast enhancement during the nephrographic-phase 


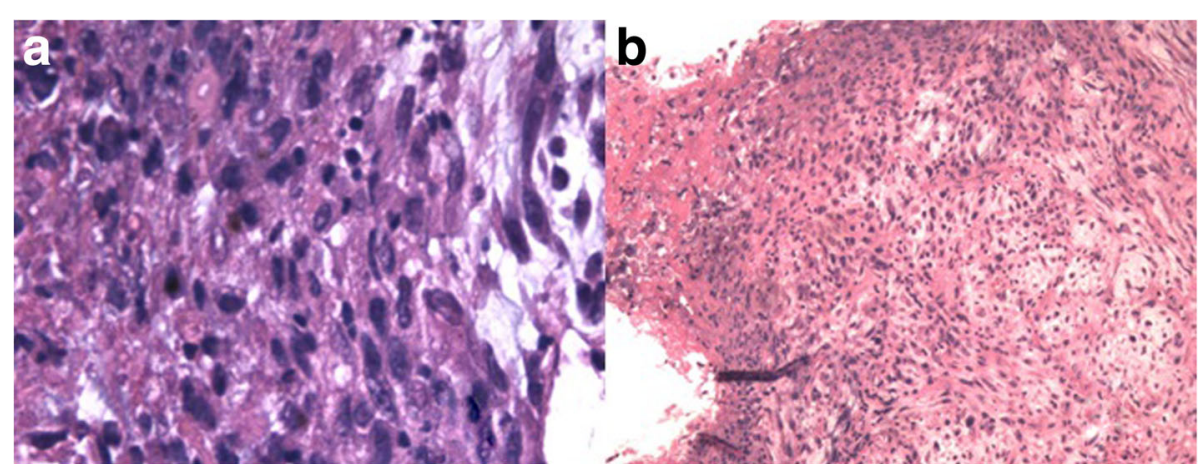

Fig. 2 Histopathologic findings. The ureteral urothelial carcinoma merges with the sarcomatoid carcinoma component, disposed in large compact aggregates. $\mathrm{HE}, \mathbf{a} \times 100, \mathbf{b} \times 400$

researchers consider that sarcomatoid carcinoma is one of the cancer metaplasia; its essence is still epithelial cancer cells which are sarcomatoid morphological differentiation. Epithelial components still express epithelial tissue markers cytokeratin, and sarcomatoid components express mesenchymal markers such as vimentin; two kinds of markers exist at the same time. The aetiology of sarcomatoid carcinoma is unclear. It may be derived from monoclonal tumour cells with epithelial and interstitial components which may be associated with 3,7,17 chromosome gene amplification and 9p21 chromosome deficiency [6]. Pathological characteristics are based on spindle cells with significant atypia, and some local tumour cells were epithelial differentiation. Most of them are high-grade urinary epithelial carcinoma. Immunohistochemistry is significantly helpful for the diagnosis, and the expression of epithelial and mesenchymal markers such as CK and vimentin is positive. And in this case, immunohistochemical features represent a typical sarcomatoid urothelial carcinoma features.

Ureteral sarcomatoid carcinoma often is found by physical examination, gross hematuria, or lower back pain. Because the illness was hidden, more than $85 \%$ of patients were found with the tumour for the T2 and T3 and accompanied by renal insufficiency. And the most of the frequent sites of metastasis were the regional lymph nodes, liver, lung and bone [7]. The present case described a woman patient who had a primary ureteral sarcomatoid carcinoma with no metastasis. The clinical symptom was just left flank pain. Imageology examination showed the hydronephrosis and occupied lesions. Compared with other cases, this one is maybe in an early stage of the tumour. The existing reliant literature is rare, and there are limited systemic options available to the best treatment option. According to the latest European Association of Urology Guidelines (2017 edition), radical nephroureterectomy remains the gold standard of the upper urinary tract urothelial carcinoma. RNU must comply with oncological principles, that is, preventing tumour seeding by avoidance of entry into the urinary tract during resection [8]. But the patient and her families refused this surgical option. In recent years, endoscopic ablation is also widely used, owing to the advantages of less trauma, less intraoperative blood loss, and protection of renal function. Compared to the upper urinary tract urothelial carcinoma, ureteral sarcomatoid urothelial

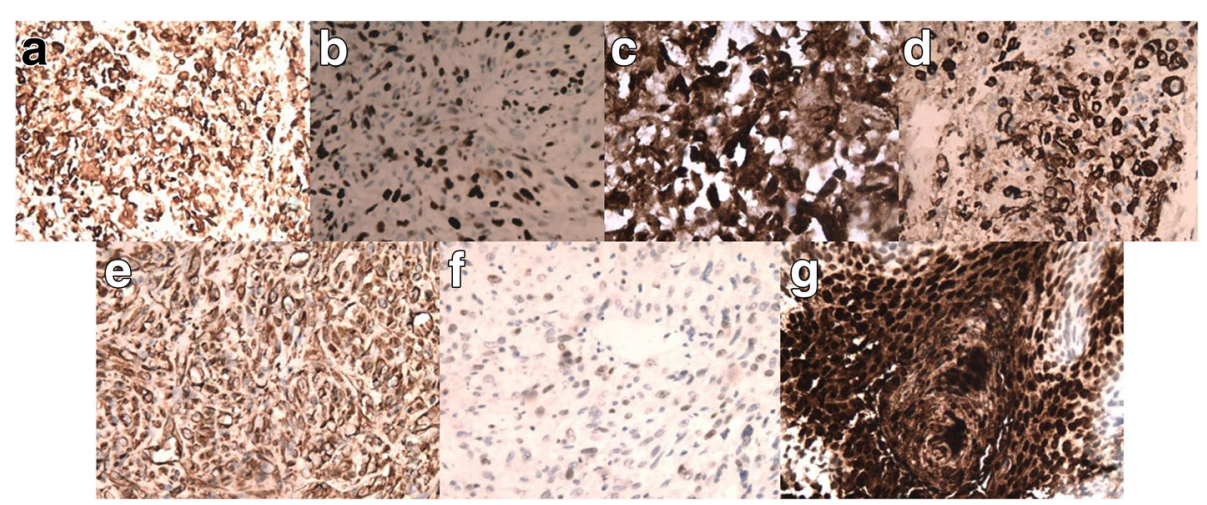

Fig. 3 Immunohistochemical staining showing positivity for cytokeratin $(\mathbf{a}, \times 200)$, carcinoembryonic antigen $(\mathbf{b}, \times 200)$, vimentin $(\mathbf{c}, \times 200)$, GATA $(\mathbf{d}, \times 200)$, CKH $(\mathbf{e}, \times 200)$, epithelial membrane antigen $(\mathbf{f}, \times 400)$ in the tumour cells. A high proliferation index of the tumour components as shown by Ki-67 reactivity $(\mathbf{g}, \times 200)$ 
carcinoma prognosis is poor and clinical guidelines is also insufficient. According to reports all over the world, ureteral sarcomatoid carcinoma lifetime is no more than 1 year [9]. According to the European urology clinical guideline, there are two major adjuvant treatment of urothelial carcinoma: (1) antegrade instillation of BCG vaccine or mitomycin $\mathrm{C}$ in the upper urinary tract by percutaneous nephrostomy and (2) retrograde instillation through a ureteric stent [8]. But the patient's basic condition cannot take the risk of the two adjuvant treatments: bleeding, ureteric obstruction, and consecutive pyelovenous influx. At the same time, the patient and her families refused the adjuvant treatment. In this case, the patient was reexamed in July of 2017, and there was not any recurrence of the tumour. It may be due to the early diagnosis and therapy.

\section{Conclusion}

In many kinds of the literature, some researchers believed the early diagnosis would contribute to a better prognosis. But according to the recently researches, there is still not a diagnosis standard in the world. Many researches found that the survival rate in kidney-sparing surgery is similar to radical nephroureterectomy in lowrisk upper urinary tract urothelial carcinoma. But the sarcomatoid urothelial carcinoma is more malignant than urothelial carcinoma. It is necessary to study and pay attention to whether there is a significant difference in survival of sarcomatoid urothelial carcinoma cases. Maybe it is essential for urologists and scientists to entirely understand the characteristics of the sarcomatoid urothelial carcinoma and make a better clinical guideline. With the development of technology and science, more and more cancerous patients' living quality and survival rate were improved.

\section{Availability of data and materials}

The datasets used and/or analysed during the current study are available from the corresponding author on reasonable request.

\section{Authors' contributions \\ $\mathrm{YW}$ and $\mathrm{HL}$ analysed and interpreted the patient data regarding its oncological features and were equal contributors in writing the manuscript. PW has given the final approval of the version to be published. All authors read and approved the final manuscript.}

Ethics approval and consent to participate Not applicable.

\section{Consent for publication}

Written informed consent was obtained from the patient for publication of this case report and accompanying images. A copy of the written consent is available for review by the Editor-in-Chief of this journal.

\section{Publisher's Note}

Springer Nature remains neutral with regard to jurisdictional claims in published maps and institutional affiliations.

Received: 27 December 2017 Accepted: 5 April 2018

Published online: 13 April 2018

\section{References}

1. Ikegami H, Iwasaki H, Ohjimi Y, et al. Sarcomatoid carcinoma of the urinary bladder: a clinicopathologic and immunohistochemical analysis of 14 patients. Hum Pathol. 2000;31(3):332-40.

2. Cheng L, Zhang S, Alexander R, Maclennan GT, Hodges KB, Harrison BT, Lopez-Beltran A, et al. Sarcomatoid carcinoma of the urinary bladder: the final common pathway of urothelial carcinoma dedifferentiation. Am J Surg Pathol. 2011;35(5):e34-46.

3. Lu W, Wang Y, Li Y, Cao Y, Han H, Zhou F. Sarcomatoid urothelial carcinoma with chondrosarcomatous differentiation of the ureter: a case report and review of the literature. Oncol Lett. 2017;13(3):1331-7.

4. Chuangzhi Z, Aiping Z, Xiangming M, Shi B, Li X. Primary adrenal sarcomatoid carcinoma metastatic to the lung: case report and review of the literature. Oncol Lett. 2016;11:3117-22.

5. Cheng L, Zhang S, Alexander R, Mac Lennan GT, Hodges KB, Harrison BT, Lopez-Beltran A, Montironi R. Sarcomatoid carcinoma of the urinary bladder: the final common pathway of urothelial carcinoma dedifferentiation. Am J Surg Pathol. 2011;35:e34-46.

6. Eble JN, Sauter G, Epstein Jl, et al. Pathology and genetics of tumors of the urinary system and male genital organs. Lyon: IARC press; 2004. p. 102-3.

7. Venyo AK-G, Titi S. Sarcomatoid variant of urothelial carcinoma (carcinosarcoma, spindle cell carcinoma):a review of the literature. ISRN UROL. 2014;2014:794563.

8. Rouprêt M, Babjuk M, Compérat E, et al. European Association of Urology guidelines on upper urinary tract urothelial carcinoma: 2017 update[]]. Eur Urol. 2018;73(1):111-22.

9. Thiel DD, Igel TC, Wu KJ. Sarcomatoid carcinoma of transitional cell origin confined to renal pelvis. Urology. 2006;67(3):622.e9-e11.

Ready to submit your research? Choose BMC and benefit from

- fast, convenient online submission

- thorough peer review by experienced researchers in your field

- rapid publication on acceptance

- support for research data, including large and complex data types

- gold Open Access which fosters wider collaboration and increased citations

- maximum visibility for your research: over $100 \mathrm{M}$ website views per year

At BMC, research is always in progress.

Learn more biomedcentral.com/submissions 